Total number of pages: 29

Total number of tables: 0

Total number of figures: 9

Total number of references: 21

\title{
Principal component analysis of the cross-axis apparent mass nonlinearity during whole-body vibration
}

\author{
Ya Huang ${ }^{1}$, Neil Ferguson ${ }^{2}$ \\ ${ }^{1}$ School of Mechanical and Design Engineering, University of Portsmouth, UK \\ ${ }^{2}$ Institute of Sound and Vibration Research, University of Southampton, UK
}

Correspondence address:

Dr Ya Huang

School of Mechanical and Design Engineering,

University of Portsmouth

Portsmouth PO1 3DJ

UK

Telephone: (+44) 02392842343

Facsimile: (+44) 02392842351

E-mail: Ya.Huang@port.ac.uk 


\begin{abstract}
During whole-body vibration (WBV), dynamic forces measured at the excitation-subject interface in directions other than the excitation axis, i.e. cross-axis response, are analysed using the principal component analysis (PCA) and virtual coherence techniques. The study applied these operations to the inline and cross-axis forces measured with twelve semisupine human subjects exposed to longitudinal horizontal nominally random vibration between 0.25 and $20 \mathrm{~Hz}$ at root mean square acceleration levels of $0.125 \mathrm{~ms}^{-2}$ and $1.0 \mathrm{~ms}^{-2}$. The source identification is realised by a reversed path, aiming to identify relative contributions and correlations between the forces in response to a single axis excitation. The inline longitudinal and the cross-axis vertical forces were found to be correlated to each other from a low (e.g. 1 to $3 \mathrm{~Hz}$ ) to a medium frequency range (e.g. 10 to $15 \mathrm{~Hz}$ ). Above this range, where the forces were much reduced, the two forces tended to be independent in their contribution to the overall response. The singular vectors and virtual coherences were able to establish the degree of correlation in each of the frequency band identified. A signal processing framework is then proposed to take into account cross-axis responses for human vibration.
\end{abstract}

Keywords: Whole-body vibration, principal component analysis, singular value decomposition

This research did not receive any specific grant from funding agencies in the public, commercial or not-for-profit sectors. 
Biomechanical responses, either forces or accelerations, in usually orthogonal directions other than the direction of excitation, i.e. cross-axis response, have been extensively reported in seated [1], standing [2] and recumbent postures [3, 4] with single-axis vertical and fore-and-aft excitations, and with simultaneous excitation in orthogonal axes $[5,6]$. Understanding the cross-axis coupling between the axis of excitation and the response axes is required for subsequently predicting body movement and forces transmitted to and through the human body undergoing vibration. Combined with the challenge to understand the mechanism causing the nonlinear magnitude dependency, in which the resonance frequency decreases with increasing excitation magnitude [7], it is imperative to quantify and separate the cross-axis response. Ordinary frequency response functions (FRFs), such as apparent mass or transmissibilities, and ordinary coherences have been regularly used to compare cross-axis responses with responses inline with the excitation [3]. However, the response in one axis could be caused by, or mathematically 'coherent to', responses in other axes. The ordinary FRF operation is not able to identify such relationships.

A preceding investigation using conditioned reverse path method was conducted to separate the cross-axis forces from the inline response force, referred to as a 'reverse path multiinput-single-output method' (reMISO) by Huang and Ferguson [8]. The method was applied to response forces measured in the inline longitudinal $(z)$, cross-axis vertical $(x)$ and crossaxis lateral $(y)$ directions. By reversing the input (longitudinal excitation acceleration) and outputs (the forces in the three axes) of the physical system [9], it is possible to assemble additional mathematical inputs from the physical output forces and mathematical constructs (e.g. square root of inline force). Depending on the specific construct, this can improve the summed multiple coherence at frequencies where the response magnitude is low. In the case studied, this is between 6 and $20 \mathrm{~Hz}$ [8]. However, for a better understanding of the nature of the nonlinearity within the cross-axis response system, an analytical process is required to quantify the relative contribution of each physical response, in this case the three axes of forces measured at the back support.

'Virtual source analysis', more commonly known as principal component analysis (PCA), assumes a new coordinate set based on the maximum variation of the data. It can be readily applied to the frequency-dependent relationship between inputs and outputs, namely the inline acceleration and the inline and cross-axis forces. By using the PCA operation alone it is usually difficult to establish relationships between the virtual and physical sources. Based on the PCA, the virtual coherence (VC) technique was developed and has been used to identify the coherences between virtual and physical sources, e.g. for an active noise cancellation system [10], and to investigate noise sources in the frequency domain around a 
diesel engine by Leclere et al. [11]. The technique effectively identified physical sources that contributed most to the virtual source over the five measurement channels.

Herein, the intention was to use the three orthogonal response forces of the semi-supine body to identify the correlation between the inline longitudinal and the cross-axis vertical forces and their frequency dependencies. PCA and the virtual coherence technique is applied to the inline and cross-axis forces measured by Huang and Griffin [3] and used previously [8]. The inline longitudinal $(\mathrm{z})$, cross-axis vertical $(\mathrm{x})$ and cross-axis lateral $(\mathrm{y})$ forces of twelve semi-supine male participants were measured at the back support while they were exposed to multiple magnitudes of random vibration in the longitudinal horizontal $(z)$ direction (Figure 1). The recumbent position was designed to minimise any forces and voluntary or involuntary movement introduced by the human subject [3]. The inline longitudinal horizontal $(z)$ and cross-axis vertical $(x)$ forces dominated the response with minimal forces in the cross-axis lateral $(y)$ direction. A drop in coherence of the inline horizontal apparent mass in the frequency range from about 10 to $16 \mathrm{~Hz}$ is present at all root mean square (r.m.s) acceleration magnitudes from 0.125 to $1.0 \mathrm{~ms}^{-2}$ r.m.s. and for all twelve subjects (see Figure 2b). From the ordinary FRFs of inline horizontal and cross-axis vertical apparent mass, and ordinary coherences, it was speculated that the reduction in 'coherent' response in the inline horizontal axis is caused by cross-axis response at those frequencies. Uncertainty on the amount of the relative contribution of the cross-axis response to the inline response exists. The source identification problem in such a context would be realised by a reversed path, where the aim is to identify contributions and correlations between the output forces in response to a single-axis excitation acceleration.

Thus the aim of the analysis is to quantify the degree of correlation established from the principal components and the virtual coherences for each physical source, so as to improve the understanding of the cross-axis coupling and their frequency dependency. It is hypothesized that the frequency dependency described by the FRFs will be determined by the excitation magnitude. As the magnitude increases, changes in the frequency range where the relationships between the inline and cross-axis responses and the acceleration change tend to shift towards a lower frequency range. The proposed PCA based identification framework will be presented on an existing dataset, allowing the value of the approach to be realised as well as providing recommendations for future processing of any human vibration measurements.

\section{Methodology}

This section starts with a brief description of how the experimental data was collected. Then subsequent processes using ordinary FRFs and coherences, the PCA formulation using 
eigen and singular value decompositions, and the virtual coherences and their implementation for the tri-axial dynamic forces are described.

\subsection{Experimental data and ordinary frequency response functions}

The analytical work presented is based on the longitudinal inline (z-axis), cross-axis vertical (x-axis) and cross-axis lateral (y-axis) forces of twelve semi-supine subjects exposed to longitudinal (z-axis) broadband random (nominally flat from 0.25 to $20 \mathrm{~Hz}$ ) vibration at 0.125 and $1.0 \mathrm{~ms}^{-2}$ r.m.s. [3]. The analysis was first demonstrated with data of one individual subject (S9) to provide a typical representation of the twelve individuals [3]. The twelve subjects, aged between 20 and 42 years, were male and mainly students and staff at the University of Southampton with median (minimum and maximum) stature $1.73 \mathrm{~m}$ (1.66 and $1.80 \mathrm{~m})$ and median total body mass $66.4 \mathrm{~kg}(58.3$ and $86.2 \mathrm{~kg})$. The experiment was approved by and followed the procedures (e.g. signed consent forms, safe exposure, etc.) required by the Human Experimentation, Safety and Ethics Committee of the Institute of Sound and Vibration Research at the University of Southampton.

A supine support comprised three parts: back support, leg rest and headrest (Figure 1). The back support was a horizontally flat rigid $660 \times 660 \times 10 \mathrm{~mm}$ aluminium plate with a high stiffness $3 \mathrm{~mm}$ thick laterally treaded rubber layer attached to the upper surface to stop any sliding. The complete back support was bolted rigidly to the upper surface of the force platform, which monitored the longitudinal forces (z-axis) and the vertical forces (x-axis) exerted by the subject on the back support. The force platform was bolted rigidly to the shaker platform. The horizontal distance between the edge of the back support and the edge of the leg rest was $50 \mathrm{~mm}$ (Figure 1). The legs of subjects rested on a horizontal flat rigid aluminium support with an 8-mm thick high stiffness rubber layer attached to the top to prevent sliding. The height of the leg rest was adjusted to allow the lower legs to rest horizontally whilst the back is also flat. The experimenters found this way of adjustment was more repeatable than using a leg-angle based approach. The headrest was a horizontal flat rigid wooden block with $75-\mathrm{mm}$ thick car-seat foam attached to the upper surface. The top surface of the complete headrest was approximately $50 \mathrm{~mm}$ higher than the back support. The horizontal distance between the back support and the headrest was adjusted by moving the headrest so that a subject's head could rest comfortably.

The forces were measured at the excitation-subject interface, or driving point, of the back support rigidly mounted on the longitudinal moving vibration platform (Figure 1). The driving point apparent masses in the inline and cross axes were computed using a cross spectral density (CSD) method, all performed in MATLAB 7.10: 


$$
H(f)=\frac{G_{F a}(f)}{G_{a a}(f)}
$$

where $H$ denotes the apparent mass; subscript ' $a$ ' the measured z-axis excitation acceleration; subscript ' $F$ ' the measured driving point forces - either the inline force $(z)$ or the cross-axis forces ( $x$ and $y$ ); $f$ the frequency in $\mathrm{Hz} ; G_{F a}(f)$ the cross spectral density (CSD) function between the response force and the excitation acceleration; $G_{a a}(f)$ the power spectral density (PSD) function of the excitation acceleration. All acceleration and force signals were acquired for 90 seconds at 200 samples per second, through an anti-aliasing filter low-passed at $67 \mathrm{~Hz}$.

\section{FIGURE 1 ABOUT HERE}

The original spectral analysis was performed with a FFT length of 2048, a Hamming window with $50 \%$ overlap (or $100 \%$ use of data) giving a frequency resolution of about $0.1 \mathrm{~Hz}$ and 36 degrees of freedom (Figure 2). In the present investigation the same data was used, but the spectral analysis was performed with a FFT length of 256, a Hamming window with $50 \%$ overlap (or $100 \%$ use of data) giving a frequency resolution of about $0.78 \mathrm{~Hz}$ and 284 degrees of freedom (following Figure 3).

The ordinary coherence function is defined as [9]:

$$
\operatorname{coh}_{a F}(f)=\frac{G_{F a}(f) G_{a F}(f)}{G_{a a}(f) G_{F F}(f)}
$$

A coherence less than unity indicates an output (e.g. a response force) that is not purely caused by a linear function of the input (e.g. the excitation acceleration), where $G_{a F}(f)$ is the complex conjugate of $G_{F a}(f)$.

\subsection{Principal components}

The principal component analysis, when applied here, assumes that the dynamic system of the human body is excited by $n$ uncorrelated principal components (PCs), or virtual sources, which is formed by a linear combination of $m$ different physically acquired sources, where $n \leq$ $m$. In the present application $m=n=3$. For $m$ measured forces and $n$ assumed virtually uncorrelated sources, the cross spectral density matrix takes the form: 


$$
G(f)=\left[\begin{array}{cccc}
G_{11}(f) & G_{12}(f) & \ldots & G_{1 n}(f) \\
G_{21}(f) & G_{22}(f) & \ldots & \\
\ldots & & & \\
G_{m 1}(f) & \ldots & & G_{m n}(f)
\end{array}\right] \text { or } \quad G(f)=\left[\begin{array}{ccc}
G_{11}(f) & G_{12}(f) & G_{13}(f) \\
G_{21}(f) & G_{22}(f) & G_{23}(f) \\
G_{31}(f) & G_{32}(f) & G_{33}(f)
\end{array}\right]
$$

where the first subscript ' 1 ' is the measured longitudinal horizontal inline response force, ' 2 ' the vertical cross-axis force, and ' 3 ' the lateral cross-axis force. So for example $G_{11}$ is the PSD for the longitudinal horizontal z-axis inline response force; $G_{12}$ is the CSD between the longitudinal horizontal $z$-axis inline response force and the vertical $x$-axis cross response force; $G_{13}$ is the CSD between the longitudinal horizontal z-axis inline response force and the lateral $y$-axis cross response force; $G_{\mathrm{ij}}$ is the complex conjugate of $G_{\mathrm{ji}}$.

For a characteristic spectral density matrix $G(f)$, one can apply an eigenvalue and eigenvector representation at each frequency such that:

$$
G(f) V(f)=D(f) V(f)
$$

The eigenvalue problem is the solution to the above equation, where $G$ in this case is an $\mathrm{m}$ by-m symmetric complex matrix. $D(f)$ is a scalar m-by-m matrix with diagonal components containing the complex eigenvalues of $G(f) . V$ is an m-by-m column vector representing the eigenvectors. In general, it is not possible to then perform an eigen decomposition of $G(f)$ by a transformation using the eigenvectors in order to produce a diagonalised matrix.

\subsection{Singular value decomposition}

For a symmetric (about its own diagonal) characteristic spectral density matrix $G(f)$, one can alternatively apply the singular value decomposition (SVD) to obtain the singular values and left and right singular vectors at each frequency:

$$
G(f)=U(f) S(f) V^{*}(f)
$$

where * denotes the complex conjugate transpose of a matrix, also called the Hermitian transpose.

The physical sources, i.e. the three response forces, can be considered as a linear combination of the principal components, comprising the positive real valued diagonal elements of $S$, and the singular vectors. $U$ is a matrix comprising columns which are the left singular vectors having the same size as $V$, a matrix with columns which are the right singular vectors. The left and right singular vectors together represent a linear relationship between the virtual and physical sources. For example, the corresponding column in $V$ and row in $U$ at each frequency $f$ for each singular value (in the diagonal of $S$ ) comprise the coefficients for the corresponding one of the three principal components (PC1, PC2 or PC3) 
which are used in the regeneration of the $G$ matrix contributions for that principal component. The number of 'significant' high-value singular values at each frequency is the number of independent contributors or principal components (Figure $3 \mathrm{a}, \mathrm{d}$ ).

The advantage of applying the SVD technique is that one can reconstruct the characteristic spectral matrix $G$ directly by multiplying the $U$ matrix, a modified $S$ matrix, and the Hermitian transposed $V$ matrix. By modifying, or indeed setting to zero certain principal components in the diagonal of the $S$ matrix, it is possible to visualise the power spectral density functions in $G$ that are contributed to by specific principal components (PC1 to PC3). For example, $G_{11}$ due to PC1 is obtained by setting PC2 and PC3 to zeros in the diagonal of the $S$ matrix, and then multiplying the $U$ matrix on its left and the transposed conjugate of the $V$ matrix on its right (Figure $3 \mathrm{~b}, \mathrm{e})$.

\section{$2.4 \quad$ Virtual coherences}

The virtual coherence identifies contribution of each virtual source, or principal component, to an individual physical source. The virtual coherence between the $j^{\text {th }}$ virtual source and the $i^{\text {th }}$ physical source is the ratio between the contribution of virtual source $j$ and the PSD of the physical source $i[9]$ :

$$
\operatorname{vcoh}_{i j}(f)=\frac{\left|U_{i j}(f)^{*} \sqrt{S_{j j}(f)}\right|^{2}}{G_{i i}(f)}
$$

where $U_{\mathrm{ij}}(f)^{*}$ is the conjugate of the left singular vector coefficient of the $i^{\text {th }}$ physical source contributing to the $j^{\text {th }}$ virtual source; $S_{\mathrm{jj}}(f)$ is the $j^{\text {th }}$ singular value of the virtual source.

\section{$3 \quad$ Results}

This section presents the results produced using the described procedures in the same order followed in the methodology section. The analysis based on a single subject (S9) is presented first and then followed by results for all twelve subjects.

\subsection{Experimental data and ordinary frequency response functions}

For the individual subject S9, the frequency ranges at which the inline force coherences drop are around 15 to $20 \mathrm{~Hz}$ at $0.125 \mathrm{~ms}^{-2}$ r.m.s. and 10 to $16 \mathrm{~Hz}$ at $1.0 \mathrm{~ms}^{-2}$ r.m.s. (Figure $2 \mathrm{~b}$ ). In this frequency range between about 10 and $18 \mathrm{~Hz}$, relatively higher coherences of the crossaxis vertical force to the excitation were observed (Figure 2d). With lower inline apparent mass (Figure 2a) compared to the cross-axis vertical apparent mass (Figure 2c) over this same frequency range, the ordinary coherences tend to suggest that the cross-axis vertical force dominates the response between 10 and $18 \mathrm{~Hz}$. In this situation it is not clear whether 
the cross-axis vertical force is correlated to the inline force, and if so by how much. The cross-axis lateral force was minimal, almost negligible, and again the ordinary coherence does not reveal much about the relative contribution of this response (Figure $2 e, f$ ).

\section{FIGURE 2 ABOUT HERE}

\subsection{Principal components and singular value decomposition}

For the individual subject $S 9$ at $0.125 \mathrm{~ms}^{-2}$ r.m.s., the inline longitudinal force dominated up to about $6 \mathrm{~Hz}$, and the cross-axis vertical force exhibited similar levels as the inline response from around 6 to $10 \mathrm{~Hz}$ and exceeded the inline force at frequencies higher than $10 \mathrm{~Hz}\left(G_{11}\right.$ and $G_{22}$ in Figure 3c). At $1.0 \mathrm{~ms}^{-2}$ r.m.s., the inline longitudinal force dominated up to about 5 $\mathrm{Hz}$, and the cross-axis vertical force had similar levels as the inline response from around 5 to $8 \mathrm{~Hz}$ and exceeded the inline from about 8 to $16 \mathrm{~Hz}\left(G_{11}\right.$ and $G_{22}$ in Figure $\left.3 f\right)$.

The first two principal components (PC1 and PC2 in Figure 3a, d) showed dominance over the frequency range up to $20 \mathrm{~Hz}$, indicating that these two independent contributors dominated the measured response spectrum but with PC1 approximately $20 \mathrm{~dB}$, i.e. a factor of 10 , more important than PC2 across the frequency range. This pattern is observed generally for the entire 12 subjects. From the median and 12 individual $P C 1$ and $G_{11}$ due to $P C 1$ in Figure $3 b$, e and Figure 4 , the inline longitudinal force $G_{11}$ appears to be the main contributor to the PC1 between about 2 and $14 \mathrm{~Hz}$ at both magnitudes. At frequencies lower than $2 \mathrm{~Hz}$ and higher than $14 \mathrm{~Hz}$, the cross-axis vertical force $\left(G_{22}\right)$ tends to govern the PC1. However, these observations are based on the qualitative rather than quantitative comparison between the PC1, the component or contribution to $G_{11}$ due to $P C 1$ and the original spectrum $G_{11}$.

\section{FIGURES 3, 4 ABOUT HERE}

A closer look at the coefficients and their difference for the linear combination of the three physical force sources contributing to PC1 confirms that at $0.125 \mathrm{~ms}^{-2}$ r.m.s. (Figure 5a) the inline response is dominant up to about $9 \mathrm{~Hz}$, and the cross-axis vertical force contributed more than the inline longitudinal force from about 9 to $20 \mathrm{~Hz}$. At $1.0 \mathrm{~ms}^{-2}$ r.m.s. (Figure $5 \mathrm{~b}$ ), the inline response is dominant up to $6 \mathrm{~Hz}$, and then the cross-axis vertical force contributed more than the inline between about 6 and $17 \mathrm{~Hz}$. Despite inter-subject variability, the frequency range for which the cross-axis vertical force contributed proportionally more than 
the inline longitudinal force decreased (i.e. approximately from 9 to $20 \mathrm{~Hz}$ to 6 to $17 \mathrm{~Hz}$ for S9, and from 9 to 20 to 10 to $16 \mathrm{~Hz}$ for the median of the twelve subjects) as the excitation magnitude increased from 0.125 to $1.0 \mathrm{~ms}^{-2}$ r.m.s. (Figure $\left.6 a, b\right)$. Such observation can be appreciated qualitatively from comparing the inline and cross-axis apparent masses and/or the PSDs at these frequency bands (Figure 2a, c and Figure 3c, f). However, the PCA operation was able to identify the virtual source, or virtual component, that yields the maximum independent variation, and then to quantify the proportional contribution, or relative importance, of each physical source to the most important virtual source in this application PC1. An alternative point of view in understanding this relationship is to consider how much each virtual source (PC) contributes to each physical source through virtual coherences.

\section{FIGURES 5, 6 ABOUT HERE}

\subsection{Virtual coherences}

For the individual subject $\mathrm{S9}$ at $0.125 \mathrm{~ms}^{-2}$ r.m.s., the virtual coherences shown in Figure $7 \mathrm{a}$ implies that the inline longitudinal force contributes primarily to the first two PCs: to PC1 it is up to about $19 \mathrm{~Hz}$, and to PC2 about 6 to $20 \mathrm{~Hz}$. In Figure 7c, the cross-axis vertical force showed the strongest influence on PC1 over the frequency range 3 to $20 \mathrm{~Hz}$, and its influence on PC2 is mainly below $3 \mathrm{~Hz}$. The difference between PC2 and PC1 virtual coherence, indicated by the bars in Figure 7, highlights the switching role between the two virtual sources in these frequency bands.

At $1.0 \mathrm{~ms}^{-2}$ r.m.s., the inline force contributed primarily to $\mathrm{PC} 1$ below $13 \mathrm{~Hz}$ and above $15 \mathrm{~Hz}$, and to PC2 between 13 and $15 \mathrm{~Hz}$ (Figure 7b). Below $10 \mathrm{~Hz}$ and above $16 \mathrm{~Hz}$, the inline force is the most important contributor. In Figure $7 d$, the cross-axis vertical force showed the strongest influence on PC1 over the frequency range 2 to $18 \mathrm{~Hz}$, with its influence on PC2 at below $2 \mathrm{~Hz}$ and above $18 \mathrm{~Hz}$.

The 'coherent' contribution of the first two physical sources to PC1 implies that the inline longitudinal force and the cross-axis vertical force are correlated to each other between about 3 and $15 \mathrm{~Hz}$ at 0.125 ms $^{-2}$ r.m.s. and between about 1 and $10 \mathrm{~Hz}$ at 1.0 ms $^{-2}$ r.m.s. At frequencies higher than about $15 \mathrm{~Hz}$ at $0.125 \mathrm{~ms}^{-2}$ r.m.s. and higher than $10 \mathrm{~Hz}$ at $1.0 \mathrm{~ms}^{-2}$ r.m.s., the two physical sources had more independent contribution to the most important principal component PC1.

Figure 6 (c, d, e, and f) illustrates the median and individual differences between virtual coherences of PC2 and PC1 to the first physical source, and between virtual coherences of 
PC2 and PC1 to the second physical source. The positive values suggest a greater contribution from the second virtual source PC2, as identifiable usually from the bars shown in Figure 7. Despite the inter-subject variability, for all twelve subjects the median differences confirm the frequency range above about $15 \mathrm{~Hz}$ at which the first and second physical sources had more independent contribution to PC1 were.

\section{FIGURE 7 ABOUT HERE}

\section{Discussion}

The principal component analysis was able to separate out independent or uncorrelated contributors of (physical) vibration sources and rank them in order. The present work adapted this source identification method in a reversed fashion where the 'output' response forces were considered as the 'sources'. The aim was to identify the relationship and relative importance of each force in relation to the reciprocal longitudinal acceleration excitation. Nevertheless, there are several limitations before the main findings can be discussed afterwards.

\subsection{Limitations}

The technique adopted in the present work cannot tell whether all of the important physical sources are included or not. For example, pitch or roll of the semi-supine body could also have influenced or been correlated to the three response forces. The fundamental assumption was that the number of physical sources acquired was larger than the number of potentially important principal components. In the original experimental study using one force plate, only the tri-axial forces were measured at the back of the supine human subjects. Moments measured about the lateral y-axis, i.e. body pitch, can be an important virtual source to analyse the biomechanical nonlinearity in the mid-sagittal plane. But this was not recorded during the original experiment [3]. Despite the effort to eliminate any voluntary body movement in the experimental setup, it is plausible that the trunk is coupled with the head via the neck, and with the lower legs via the knees with some degree of involuntary movement. Therefore forces and moments measured underneath the head and the lower legs could help interpret the motion transmission paths. However, no force plate was mounted at these two locations. Increased dynamic discomfort has been shown to relate to the angular velocity between the neck and the trunk during supine transportation [12]. Measurements of angular motion or moment could be important physical sources. 
The current work draws on the following three areas of analysing the human biomechanical data during multiple channel measurements.

\subsection{Virtual coherence and multiple coherence}

The analysis was performed with all twelve subjects whose apparent masses were originally reported [3]. The presented results from an individual and the twelve subjects showed typical characteristics of the relative contributions in each axis. In general, the inline longitudinal and the cross-axis vertical forces were correlated to each other from a low (e.g. $3 \mathrm{~Hz}$ at $0.125 \mathrm{~ms}^{-}$ 2 r.m.s. and $1 \mathrm{~Hz}$ at $1.0 \mathrm{~ms}^{-2}$ r.m.s.) to a medium frequency (e.g. $15 \mathrm{~Hz}$ at $0.125 \mathrm{~ms}^{-2}$ r.m.s. and $10 \mathrm{~Hz}$ at $1.0 \mathrm{~ms}^{-2}$ r.m.s.). Above the medium frequency where the apparent mass is much reduced compared to lower frequencies, the two forces tended to be independent in their contribution to the overall response. The exact frequency at which these bands switch varied, but the twelve subjects all conformed to the pattern identified by the above bands (Figure 6). This was consistent with the multiple coherence analysis performed with the same group of subjects using conditioned reverse path method (reMISO), where after removing 'correlated' contributions of cross-axis forces the inline force dominated below $10 \mathrm{~Hz}$ at 1.0 $\mathrm{ms}^{-2}$ r.m.s. [8]. This conditioned reverse path FRF unveiled that an uncorrelated 'linear' relationship between a physical input (acceleration) and the outputs (inline and cross-axis forces) had greater variation around the primary resonance frequency, i.e. between 0.5 and 5 $\mathrm{Hz}$. However, the multiple coherence could not quantify the linear combination of the coefficients identified by the singular vectors of the dominant principal components (Figure 5 and 6). With the help of the singular vector coefficients and virtual coherences, one can rank and compare according to the relative importance of each physical source.

The previous reMISO investigation offered specific construct of mathematical 'inputs' as substitutes to the physical sources - a technique used to improve the summed multiple coherence at frequencies where the response magnitude is low [8]. In the present case this is between 6 and $20 \mathrm{~Hz}$. Accompanied (centred) skewness and kurtosis analysis of the response forces suggested that there were potential anatomical 'end-stops' for the sprung mass in the inline axis with potentially different coupling mechanisms present at different excitation magnitude. To analytically model these speculated mechanisms one needs to quantify the proportionality of the relative influences from each physical source in this frequency range. The virtual coherences are able to illustrate the interchange of the inline and cross-axis forces in contributing to the first and second PCs (Figure 7).

\subsection{Magnitude dependent biomechanical nonlinearity}

Despite the posture and positions of a sitting, standing or recumbent human occupant, an increase in WBV excitation magnitude causes a decrease in the primary resonance 
frequency $[1,2,3,4]$. This is true with different single axis excitation and simultaneous multiple axes excitation $[5,6]$. If the human-excitation interface is modelled as a rigid moving base coupled with translational and rotational springs and dampers, representing the soft tissue and geometric arrangement at the interface [13], and then connected to a 'sprung mass', representing the inertia of the skeletal structure and main body mass away from the interface, it is possible to stipulate from previous studies that increased relative movement between the base and the sprung mass will increase the nonlinear magnitude dependency. As the excitation magnitude increases the restoring forces do not increase in a proportional manner, compared to a lower magnitude input, implying that the body system is 'more nonlinear' at higher magnitudes.

The increase in the magnitude dependent nonlinearity can come in the forms of added axes of simultaneous excitation, changing direction of excitation, where arguably some axes could be more nonlinear than the others, and directly increase in excitation magnitude. All of these factors can increase the cross-axis response which in term affect the magnitude dependency seen in the transfer functions of each motion transmission path. For example, additional axes of simultaneous vibration decreased the apparent mass resonance frequency and increase cross-axis response of seated occupants $[5,6]$. Specifically the current analysis shows that the inline and the cross-axis responses to a single axis of excitation dominate different frequency bands. The cross-axis vertical response dominates 9 to $20 \mathrm{~Hz}$ at $0.125 \mathrm{~ms}^{-2}$ r.m.s. and 6 to $17 \mathrm{~Hz}$ at $1.0 \mathrm{~ms}^{-2}$ r.m.s. for S9 (Figure 5) - the frequency band overlapped with an apparent drop in the inline coherence function (Figure $2 b$ ). In the past, the observation has been that the frequency of the coherence drop decreases with increasing excitation magnitude [3]. In fact the underlying reason is the increased cross-axis coupling demonstrated in proportion to the singular vectors for the dominant virtual sources, i.e. PC1 and PC2.

The source of cross-axis coupling in whole-body vibration was previously speculated to be the geometric arrangement of the skeletal structure and the soft tissue at the excitationsubject interface $[14,15]$. With the recumbent position and an excitation in the shearing direction of the soft tissue at the contact interface, the vertical cross-axis response would not be primarily caused by bending of the spine nor pitching of the pelvis. Instead it was more likely to be caused by the 'weight transfer' of the sprung mass, i.e. the bony and soft constructions of the body above the interface soft tissue. The linear combinations of the singular vector coefficients identified by the PCA operation would assist in the development of analytical models like this. By tuning the model viscoelastic and geometric parameters, it is plausible that a plane kinematic-kinetic model comprised of the two translational axes and one orthogonal rotational axis could represent the dominant primary resonance at below 5 
$\mathrm{Hz}$ and the cross-axis coupling at higher frequencies in the present work [13]. Such a model will be able to interpret the inline and cross-axis responses of the human-excitation interface, and the associated coherence drop seen in the ordinary frequency responses functions. However, the cause of the magnitude dependent nonlinearity remains unclear.

In the past decade, most experimental evidence of the cause of the magnitude dependent nonlinearity have pointed to the shift of the dynamic characteristics of the soft tissue at the excitation-subject interface [3, 16]. Huang et al. [17] found a similar dynamic property shift in freshly sacrificed porcine skeletal muscle tissues when excited by different magnitudes of impulsive impact hammer loadings. The skeletal muscles are a complex matrix of viscoelastic structures intertwined with multiple layers of fluids. A numerical model with anatomical representation of the soft tissue of such microscopic and finite framework will be complicated, but will not necessarily guarantee a 'macro' level magnitude dependent nonlinear response observed in the frequency response functions at the excitation-subject interface. On the other hand, designs of vibration isolation equipment for human occupants require a reliable quantification tool to measure the human body dynamic responses at varying magnitudes. So far, to quantify the magnitude dependency a single resonance frequency is extracted from the FRFs - either using the original transfer function, or 'calibrating' a lumped parameter model to fit specific FRFs, be it a transmissibility or an apparent mass. A non-parametric statistic tool such as Wilcoxon matched-pairs signed ranks test can then be used to tell if there is a significant difference between resonance frequencies at two different excitation magnitudes. This approach is difficult to quantify the size of the difference between two resonance frequencies due to a number of factors: the frequency resolution could be too coarse such that any difference especially at lower frequencies, e.g. below $10 \mathrm{~Hz}$ for WBV, would have been smeared out; multiple local maxima could skew the identification of the global resonance frequency. Given the highly damped resonance of the human body system, a single resonance frequency can hardly represent the frequency band occupied by the usually broad resonance. A mathematical tool is required to summarise the characteristics of the shifting frequency band due to changes in excitation magnitude.

By mathematically integrating the PSD of the inline force and that generated using PC1 and then normalising by dividing the integral at the highest frequency of interest, i.e. $20 \mathrm{~Hz}$ for WBV, the shape and area between the two integral curves at the two different excitation magnitudes reveal the difference of the contributions to the root mean square (r.m.s.) response over the entire frequency range (Figure 8 ). The cumulative PSDs for $G_{11}$ and due to PC1 are normalised and defined as: 


$$
\begin{gathered}
c P S D_{G_{1 I}}(f)=\frac{\sum_{f=0}^{20} G_{I I}(f)}{G_{I I}(20)} \\
c P S D_{P C l}(f)=\frac{\sum_{f=0}^{20} P C l(f)}{P C l(20)}
\end{gathered}
$$

where $\mathrm{PC} 1$ is the PSD due to the $\mathrm{PC} 1$ at frequency $f$ in $\mathrm{Hz}$; '20' in $\mathrm{Hz}$ is the highest frequency of the analysis. The differences between two excitation magnitudes for $G_{11}$ and PC1 are obtained by:

$$
\begin{gathered}
\Delta c P S D_{G_{I I}}=\sum_{f=0}^{20}\left(c P S D_{G_{1 I} \text { high }}(f)-c P S D_{G_{1 I} \text { low }}(f)\right) \\
\Delta c P S D_{P C l}=\sum_{f=0}^{20}\left(c P S D_{P C 1 \_h i g h}(f)-c P S D_{P C 1 \_l o w}(f)\right)
\end{gathered}
$$

where the subscript 'high' indicates the cumulative PSD at $1.0 \mathrm{~ms}^{-2}$ r.m.s., and the 'low' for $0.125 \mathrm{~ms}^{-2}$ r.m.s.

\section{FIGURE 8 ABOUT HERE}

For the first physical source $\left(G_{11}\right), \triangle c P S D_{G_{11}}$ indicates the area between the high and low magnitude cumulative PSD curves in Figure 8a. If a single primary resonance dominates the frequency range of interest $[3,4$, and 16$]$, this difference in the cumulative PSD is able to quantify the difference due to excitation magnitude. For S9 shown, this is $1.35-$ a dimensionless quantity where both the minuend and the subtrahend are the cumulative PSDs normalised at $20 \mathrm{~Hz}$. Nevertheless, this difference does not capture the shape of the enclosed area formed by the two normalised cumulative PSD curves. A way to describe the shape as a function could quantify the nonlinear magnitude dependency. This can be achieved by scaling, translating and rotating a hyperbolic tangent function. Worden et al. [18] demonstrated this approach to construct a nonlinear analytical model for the restoring damping force-relative velocity relationship of an automotive shock absorber. The application of this technique lies outside the scope of the current investigation.

For the first virtual source (PC1), $\triangle c P S D_{P C 1}$ indicates the area between the high and low magnitude cumulative PSD curves in Figure 8b. A crossing between the low and high magnitudes cumulative PSD curves indicates a more dramatic change in gradient of the $c P S D_{P C 1}$ at the lower magnitude. For S9, this crossing between the low and high magnitude $c P S D_{P C 1}$ is at $2.34 \mathrm{~Hz}$. This change of gradient coincides with the frequency band below 2 to 
$3 \mathrm{~Hz}$ where the singular vectors at the lower magnitude were dominated by the cross-axis vertical force in Figure $5 a$ for 59 with the bars showing the difference in the singular vectors between the cross and inline forces and in Figure 6a for the 12 subjects showing only the difference represented by the bars as in Figure $5 \mathrm{a}$. This dominance was switched to the inline longitudinal force right above this frequency band.

\subsection{Multi-channel biomechanical signal processing for whole-body vibration}

The present analysis demonstrated a relatively simple example with only three different physical sources that have high, medium and low relative contributions to the overall dominant virtual response i.e. PC1. With accelerations and transmissibilities measured in multiple translational and rotational directions between the base of excitation and a location on the body or between two locations on the body, one can derive the correlation between the response axes and their relative contributions. Ordinary FRFs of such physical measurements were able to identify frequency bands of amplified vibration for supine casualties wearing board litters and neck collars during transportation [19]. A comparison between the principal components and virtual coherences of these physical vibration sources obtained from single-axis excitation and multi-axis excitation could provide a more quantitative insight into the effect of additional axes of combined excitations on individual responses. The signal processing workflow adopted in the current research and proposed is summarized in Figure 9.

\section{FIGURE 9 ABOUT HERE}

The characterisation and quantification of the cross-axis response and excitation magnitude dependent nonlinearity of the seat-occupant assembly are at the centre of devising any mitigation systems for shock and whole-body vibration. These systems vary widely in their form factor and application, be it a compressed foam-seat frame unit seen in most automotive and rail industry, a suspended module of single or multi-axial movement, a multiaxial suspended cabin system, or just a cushion found on off-road vehicles, earth-moving machineries and fast marine crafts. Numerical models utilising multi-body dynamics module or even containing finite elements offers detailed depiction of vibration modes of the human body with a degree of anatomical representation [20]. However, they come at a cost of 1) usually much simplified boundary conditions and restraints which render the model unrealistic with large variation of excitation magnitude; 2) dependence on the fixture of a particular posture and body position which is rarely guaranteed in reality; 3) relying on largely linear dynamic components and nonlinear geometric arrangement which are not aligned with the mechanistic understanding of the magnitude dependent nonlinear behaviour. For multiaxial excitation and multi-axial response WBV measurement and investigation, it is 
recommended that first the analysis could apply the PCA method presented here to quantify relative contribution of cross-axis responses and their corresponding frequency bands, and then the reMISO approach [8] would provide further insights into the linearised transfer functions using conditioned inputs. The latter have the advantage of providing a potential mathematical formulation using the physical sources that serve as analytical solutions to the nonlinearity observed. Their simplicity and allowance for 'loose' assumptions of the test subjects and boundary conditions may suit a wider range of applications at a more acceptable cost.

An alternative approach kernel principal component analysis (KPCA) maps the input data into a high-dimensional feature space via a nonlinear mapping first and then performs a linear PCA in the same feature space. Although nonlinear feature mapping is not the primary objective of the present study, the KPCA method offers a possible comparison with the reMISO approach [8], where nonlinear formulations of the physical outputs or virtual sources can be analysed to 'recover' low coherences. KPCA has been extensively applied for diagnosis and health feature extraction, for example rotating machines [21], usually only with response data statistics and not time synchronised. Typically there is no unique input or excitation measurement to obtain any transfer function measurement. For human response to vibration studies, the physical inputs are usually measured in sync with the outputs to establish the frequency responses with both modulus and phase information. For this reason, a linear mapping of the feature space is preferred to establish the proportional contribution of each virtual source at each frequency first, and then a nonlinear analytical approach, be it reMISO or KPCA, could be deployed to further inspect the pattern of coupling between sources at certain frequency bands.

\section{$5 \quad$ Conclusions}

A frequency response function based principal component analysis was demonstrated on a relatively simple example of three acquired biomechanical dynamic forces, which contributed in different proportions to the dominant virtual sources or principal components. The PCA process was able to identify frequency ranges where the inline and vertical cross-axis forces are correlated or uncorrelated to each other. It also identified the degree of correlation from the singular vectors of the principal components and the virtual coherences for each physical source. The corresponding eigenvector of the first principal component (PC1) changed in the mid frequency band, indicating a shift in dominance moving from the inline longitudinal to the vertical cross-axis force. The frequency bands in which the PC1 and PC2 contributed most decrease with increasing excitation magnitude, providing a different quantification tool for the biomechanical magnitude dependency. 


\section{References}

[1] N. Nawayseh, M.J. Griffin, Non-linear dual-axis biodynamic response to vertical wholebody vibration, J. Sound Vibration 268 (2003) 503-523.

[2] G.H.M.J. Subashi, Y. Matsumoto, M.J. Griffin, Apparent mass and cross-axis apparent mass of standing subjects during exposure to vertical whole-body vibration, J. Sound Vibration 293 (2006) 78-95.

[3] Y. Huang, M.J. Griffin, Nonlinear dual-axis biodynamic response of the supine human body during longitudinal horizontal whole-body vibration, J. Sound Vibration 312 (2008) 273295.

[4] Y. Huang, M.J. Griffin, Nonlinear dual-axis biodynamic response of the supine human body during vertical whole-body vibration, J. Sound Vibration 312 (2008) 296-315.

[5] G. Zheng, Y. Qiu, M.J. Griffin, Vertical and dual-axis vibration of the seated human body: Nonlinearity, cross-axis coupling, and associations between resonances in transmissibility and apparent mass, J Sound Vibration 331 (2012) 5880-5894.

[6] N.J. Mansfield, S. Maeda, Comparison of the apparent masses and cross-axis apparent masses of seated humans exposed to single- and dual-axis whole-body vibration, J. Sound Vibration, 298 (2006) 841-853.

[7] Y. Huang T.P. Gunston, Review of human perception of whole-body vibration and mechanical shock: implications of biodynamic nonlinearities and soft tissue. Proceedings of the $44^{\text {th }}$ United Kingdom Conference on Human Responses to Vibration, University of Loughborough, Leicestershire, England, 2009.

[8] Y. Huang, N.S. Ferguson, Identification of biomechanical nonlinearity in whole-body vibration using a reverse path multi-input-single-output method, J. Sound Vibration 419 (2018) 337-351.

[9] J.S. Bendat, A.G. Piersol, Engineering Applications of Correlation and Spectral Analysis, $2^{\text {nd }}$ Edition, John Wiley \& Sons, Inc. 1993.

[10] S.M. Price, R.J. Bernhard, Virtual coherence: a digital processing technique for 
incoherent source identification. In: Proceedings of IMAC 4 (1986) NY, USA.

[11] Q. Leclere, C. Pezerat, B. Laulagnet, L. Polac, Application of multi-channel spectral analysis to identify the source of a noise amplitude modulation in a diesel engine operating at idle, Applied Acoustics 66 (2005) 779-798.

[12] J. DeShaw, S. Rahmatalla, Predictive discomfort of supine humans in whole-body vibration and shock environments, Ergonomics (2016) 59(4) 568-581.

[13] N. Nawayseh, M.J. Griffin, A model of the vertical apparent mass and the fore-and-aft cross-axis apparent mass of the human body during vertical whole-body vibration, J. Sound Vibration, 319 (2009) 719-730.

[14] S. Kitazaki, M.J. Griffin, Resonance behaviour of the seated human body and effects of posture. Journal of Biomechanics 31 (1998) 143-149.

[15] Y. Matsumoto, M.J. Griffin, Movement of the upper-body of seated subjects exposed to vertical whole-body vibration at the principal resonance frequency, J. Sound Vibration 215 (1998) 743-762.

[16] Y. Huang, M.J. Griffin, Nonlinearity in apparent mass and transmissibility of the supine human body during vertical whole-body vibration, J. Sound Vibration 324 (2009) 4292-452.

[17] Y. Huang, M. Takaza, C. Simms, Dynamics characterisation of passive porcine skeletal muscle using impact hammer: inline responses. In, Proceedings of the 50th UK Conference on Human Responses to Vibration, ISVR, University of Southampton, Southampton, England, 2015

[18] K. Worden, D. Hickey, M. Haroon, D.E. Adams, Nonlinear system identification of automotive dampers: A time and frequency-domain analysis. Mechanical Systems and Signal Processing, 23 (2009) 104-126.

[19] J. Meusch, S. Rahmatalla, Whole-body vibration transmissibility in supine humans: Effects of board litter and neck collar, Applied Ergonomics 45 (2014) 677-685.

[20] M.M. Verver, J. van Hoof, C.W.J. Oomens, N. van de Wouw, J.S.H.M. Wismans, Estimation of spinal loading in vertical vibrations by numerical simulation. Clinical Biomechanics 18 (2003) 800-811. 
[21] Q. He, F. Kong, R. Yan, Subspace-based gearbox condition monitoring by kernel principal component analysis, Mechanical Systems and Signal Processing 21 (2007) 17551772. 


\section{Figure captions}

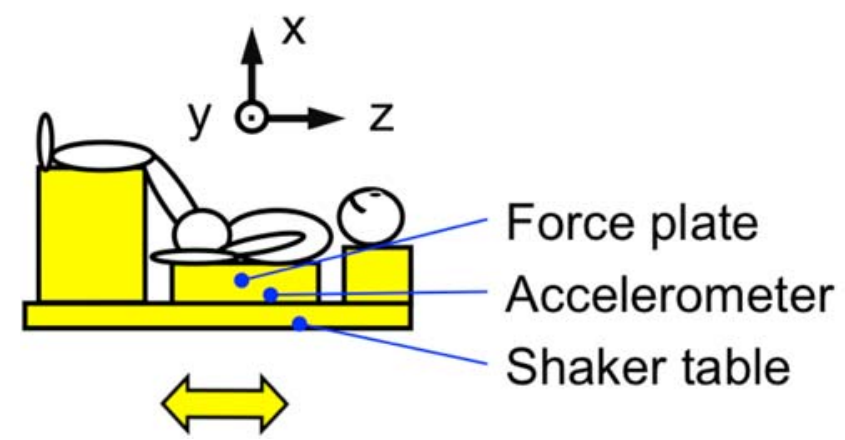

Figure 1 The schematic shows the typical semi-supine position adopted for all test subjects exposed to longitudinal (z-axis) nominally flat random vibration between 0.25 and 20 $\mathrm{Hz}$. Forces in the inline longitudinal (z-axis), cross-axis vertical (x-axis), and cross-axis lateral $(y$-axis) directions were measured to calculate the apparent masses [3]. 

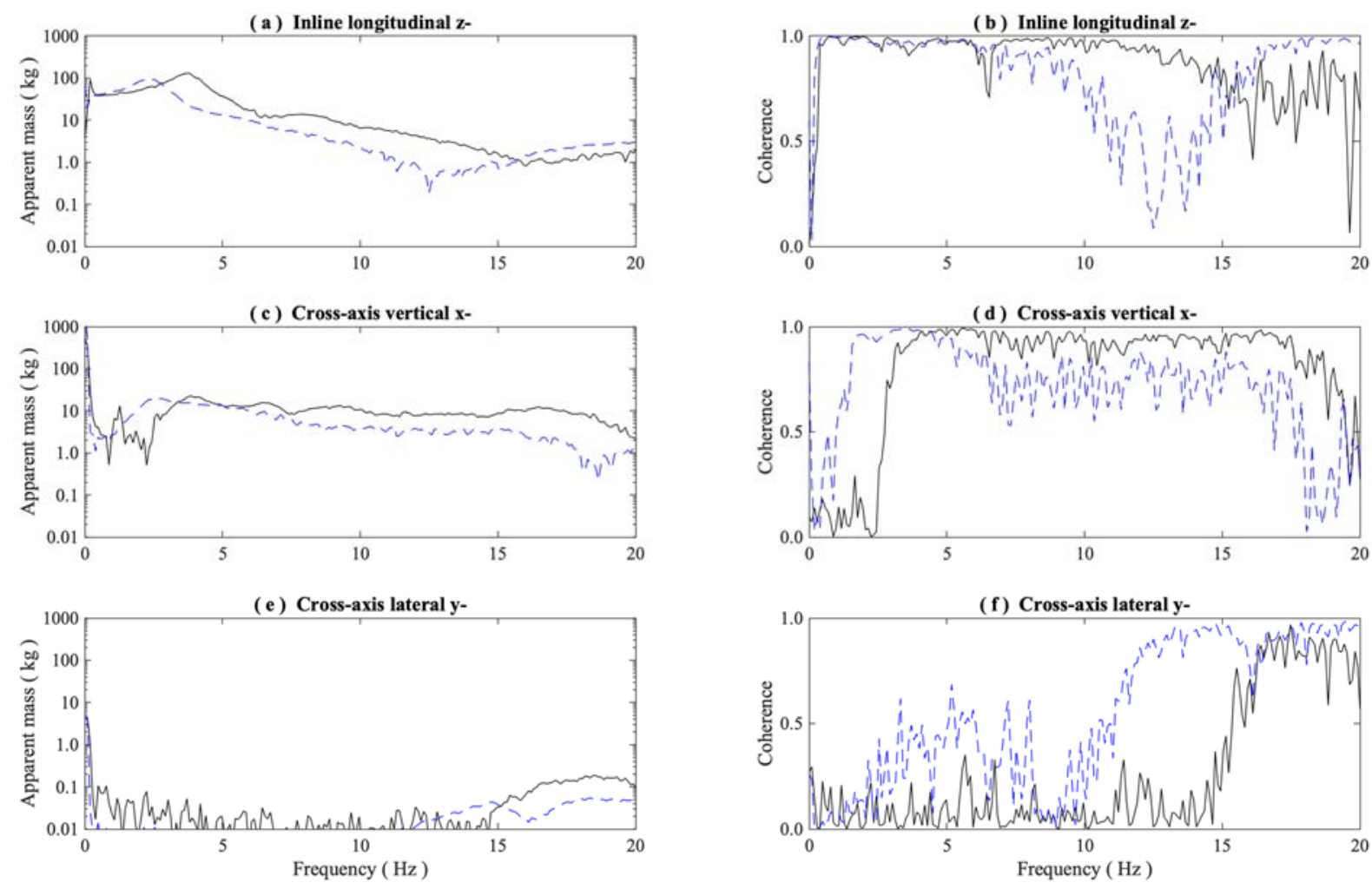

Figure 2 Individual (S9) inline longitudinal (z-axis) apparent mass peaked at $2.3 \mathrm{~Hz}$ and $98.8 \mathrm{~kg}$ (at $1.0 \mathrm{~ms}^{-2}$ r.m.s., broken line) and at $3.8 \mathrm{~Hz}$ and $130.8 \mathrm{~kg}$ (at $0.125 \mathrm{~ms}^{-2}$ r.m.s., solid line) (a) and their coherences (b); cross-axis vertical (x-axis) apparent mass peaked at $2.5 \mathrm{~Hz}$ and $20.9 \mathrm{~kg}$ (at $1.0 \mathrm{~ms}^{-2}$ r.m.s., broken line) and at $3.8 \mathrm{~Hz}$ and $22.6 \mathrm{~kg}$ (at $0.125 \mathrm{~ms}^{-2}$ r.m.s., solid line) (c) and their coherences (d); and cross-axis lateral (y-axis) apparent mass at 1.0 (broken line) and at $0.125 \mathrm{~ms}^{-2}$ r.m.s. (solid line) (e) and their coherences (f). 


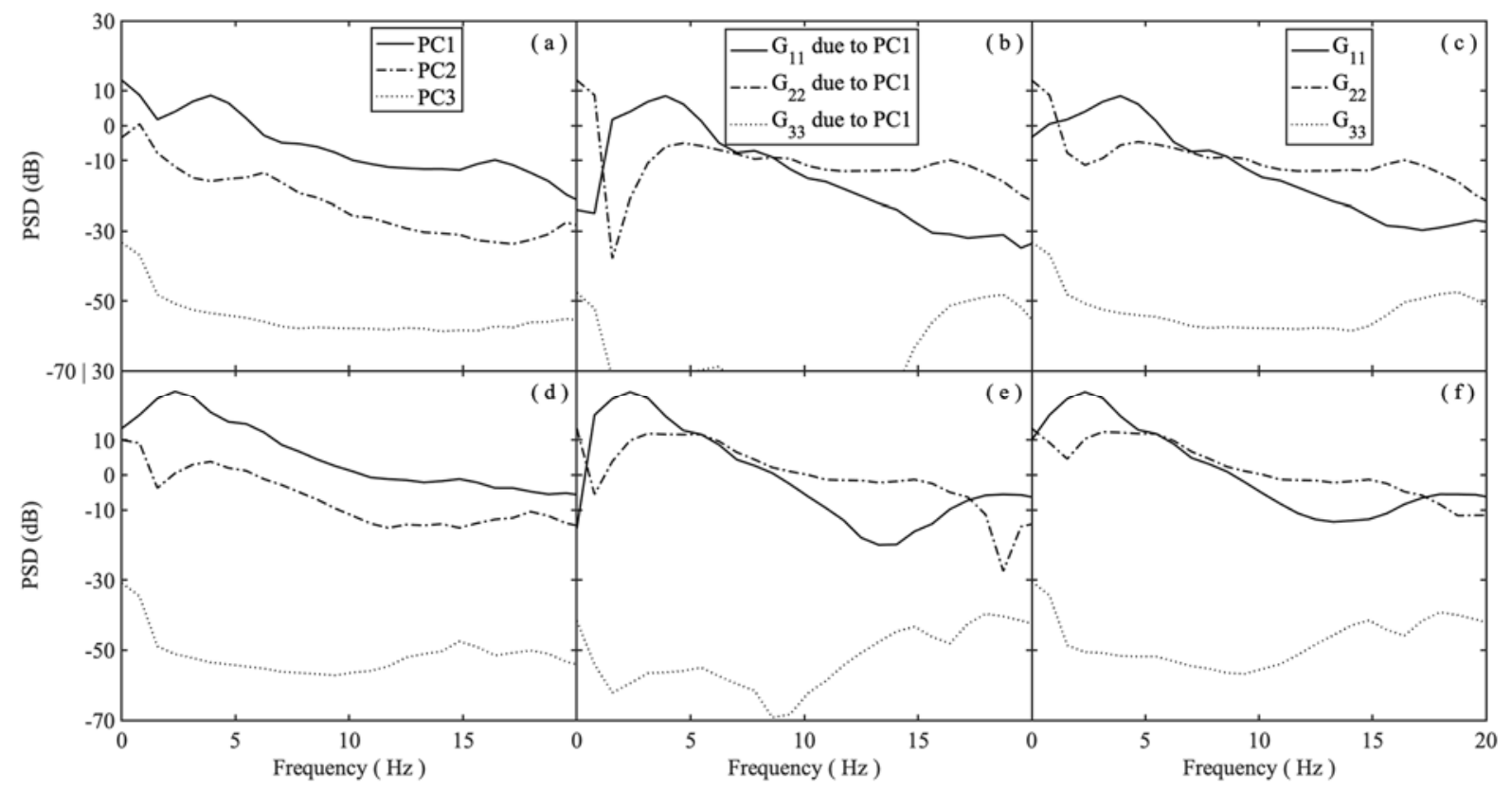

Figure 3 Individual (S9) relative level of the power spectral density functions of the three principal components (PC1: solid line; PC2: dash-dot line; PC3: dotted line) at 0.125 (a) and $1.0 \mathrm{~ms}^{-2}$ r.m.s. (d); the PSD of the inline longitudinal force due to the first principal component ( $G_{11} P C$ 1: solid line; $G_{22} P C 1$ : dash-dot line; $G_{33} P C 1$ : dotted line) at 0.125 (b) and $1.0 \mathrm{~ms}^{-2}$ r.m.s. (e); the PSD of the inline longitudinal force $\left(G_{11}\right.$ : solid line; $G_{22}$ : dash-dot line; $G_{33}$ : dotted line) at 0.125 (c) and $1.0 \mathrm{~ms}^{-2}$ r.m.s. (f). 


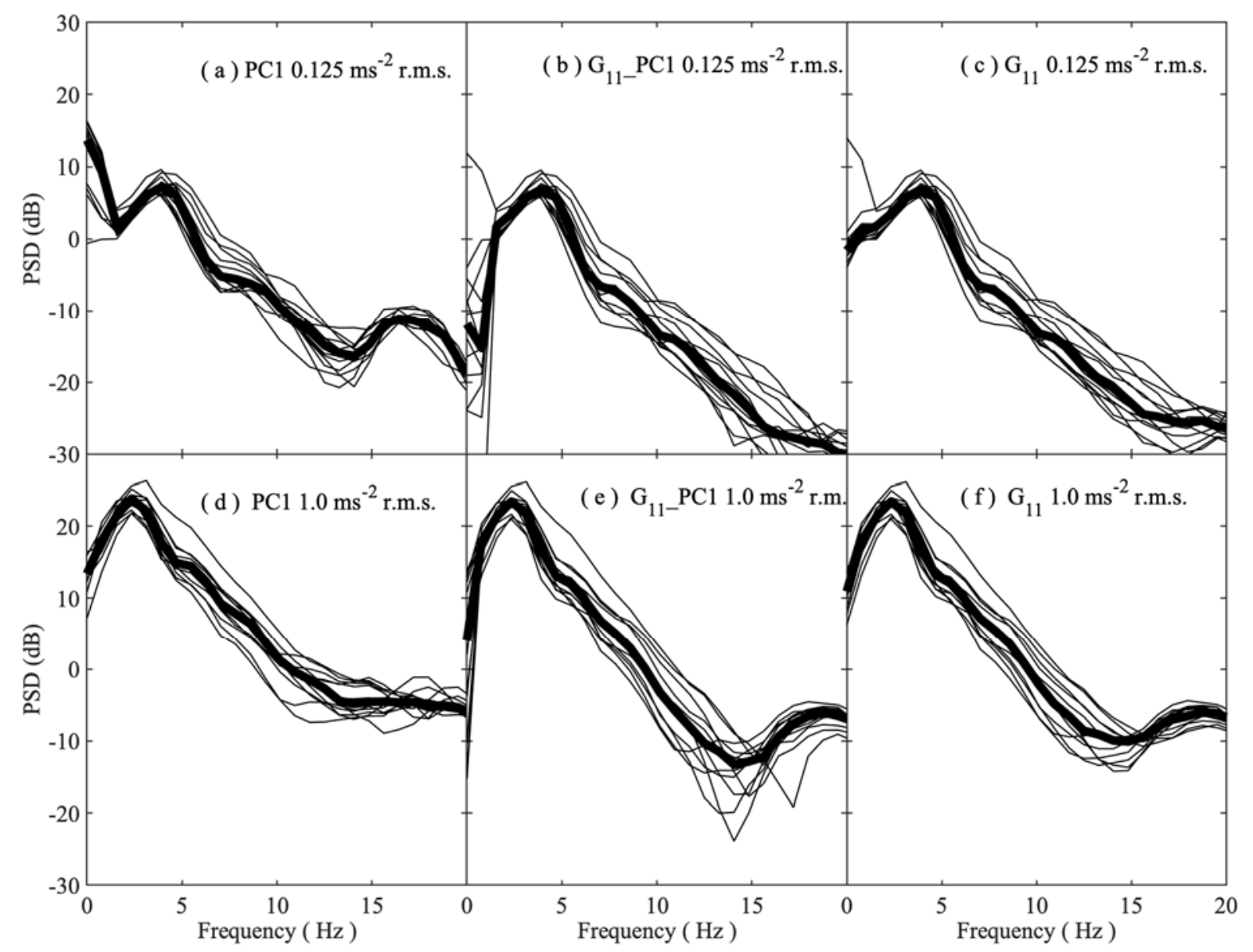

Figure 4 Median (thick lines) and individual (thin lines) relative level of the power spectral density functions of the 12 subjects: the first principal component (PC1) at 0.125 (a) and $1.0 \mathrm{~ms}^{-2}$ r.m.s. (d); the PSD of the inline longitudinal force due to the first principal component $\left(G_{11}\right.$ PC1) at 0.125 (b) and $1.0 \mathrm{~ms}^{-2}$ r.m.s. (e); the PSD of the inline longitudinal force $\left(G_{11}\right)$ at 0.125 (c) and $1.0 \mathrm{~ms}^{-2}$ r.m.s. (f). 


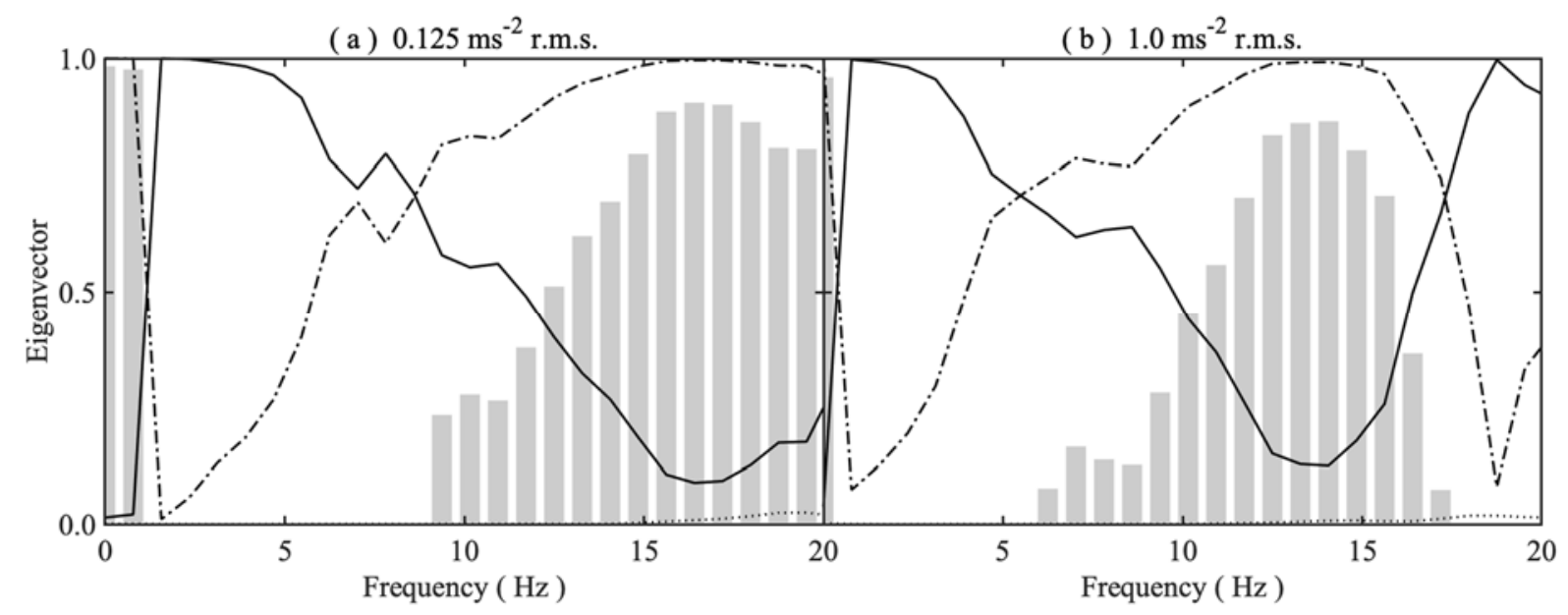

Figure 5 Individual (S9) absolute values of singular vectors of the inline longitudinal force (solid line), cross-axis vertical force (dash-dot line), and cross-axis lateral force (dotted line) to the first principal component (PC1) showing the unified (to one) linear coefficients of the physical sources that contributed to PC1 at 0.125 (a) and $1.0 \mathrm{~ms}^{-2}$ r.m.s. (b). The bars show the difference between the second physical source (i.e. cross-axis vertical force) and the first physical source (inline longitudinal force), with positive values implying a greater contribution from the second physical source. 


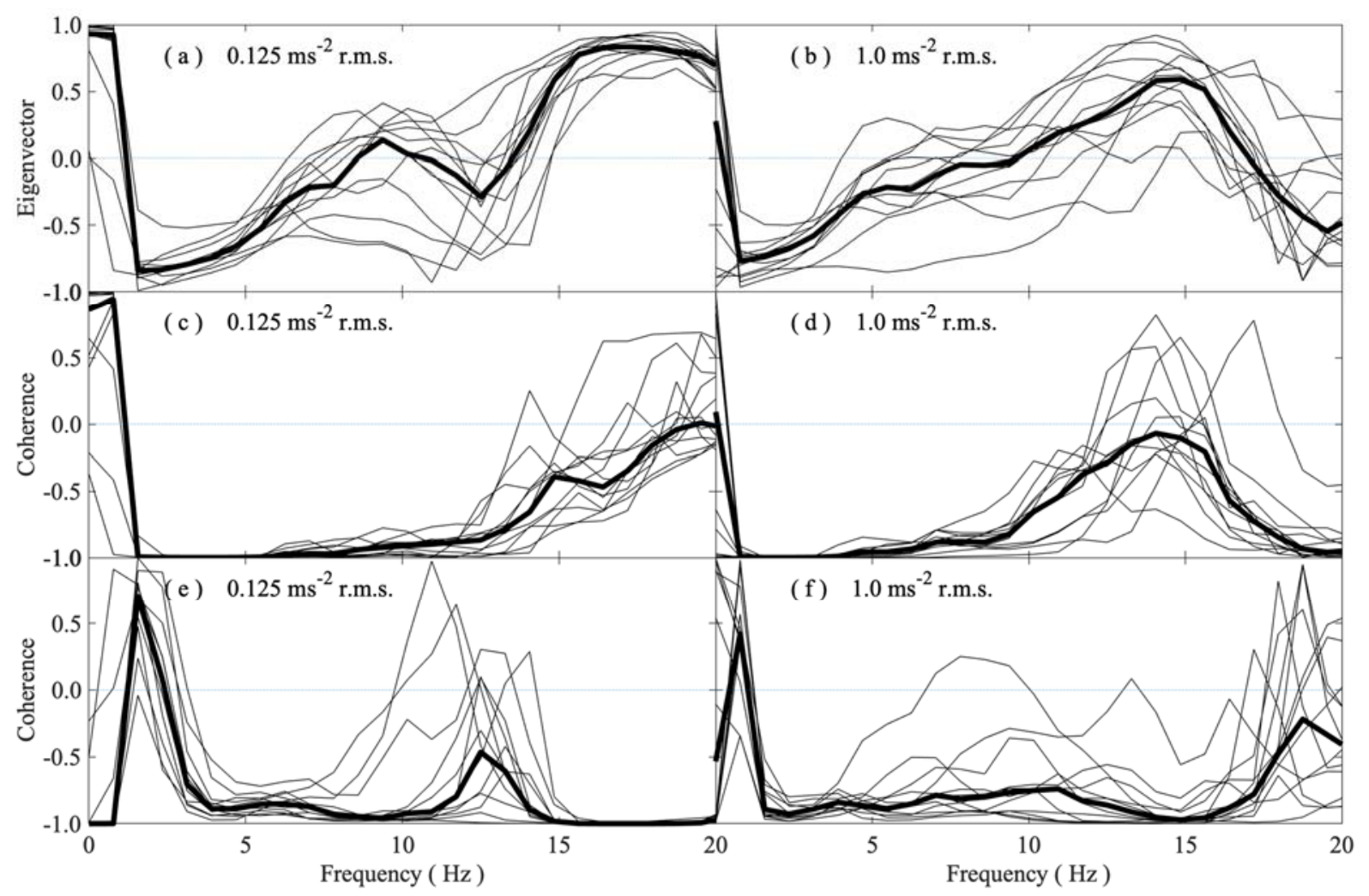

Figure 6 Differences between singular vectors of the second physical source (i.e. cross-axis vertical force) and the first physical source (inline longitudinal force) at 0.125 (a) and at $1.0 \mathrm{~ms}^{-2}$ r.m.s. (b) with median (thick lines) and individual (thin lines) data of 12 subjects. Differences between virtual coherences of PC2 and PC1 to the first physical source at 0.125 (c) and at $1.0 \mathrm{~ms}^{-2}$ r.m.s. (d) with median (thick lines) and individual (thin lines) data of 12 subject. Differences between virtual coherences of PC2 and PC1 to the second physical source at 0.125 (e) and at $1.0 \mathrm{~ms}^{-2}$ r.m.s. (f) with median (thick lines) and individual (thin lines) data of 12 subjects. 


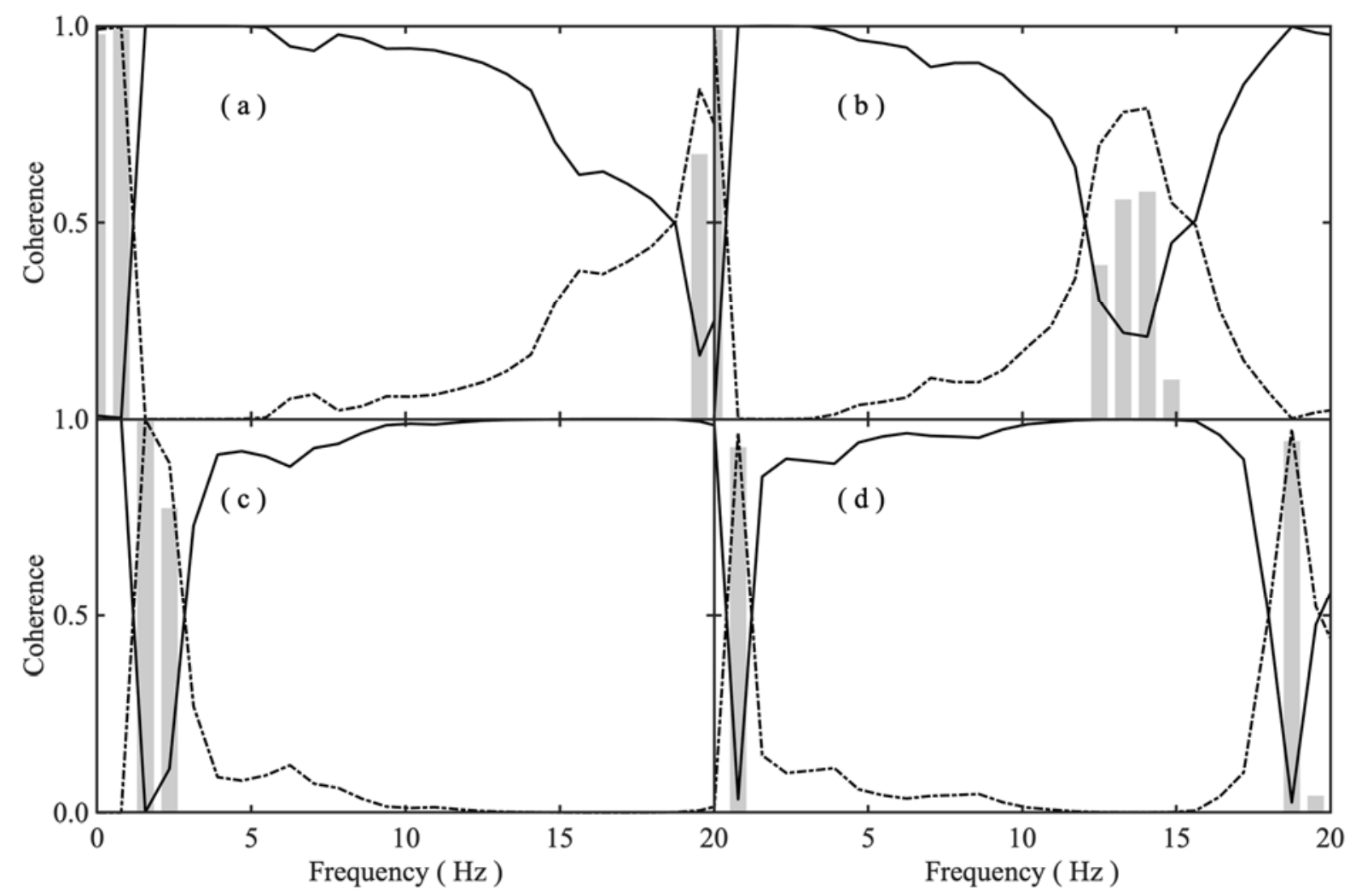

Figure $7 \quad$ Individual (S9) virtual coherences of the principal component PC1 (solid line) and PC2 (dash-dot line) to the first physical source (inline longitudinal force) at 0.125 (a) and $1.0 \mathrm{~ms}^{-2}$ r.m.s. (b), and virtual coherences of PC1 and PC2 to the second physical source (cross-axis vertical force) at 0.125 (c) and $1.0 \mathrm{~ms}^{-2}$ r.m.s. (d) showing the linear contribution of each PC to the physical sources. The bars show the difference between virtual coherences of PC2 and PC1, with positive values implying a greater contribution from the second virtual source PC2. 
(a)

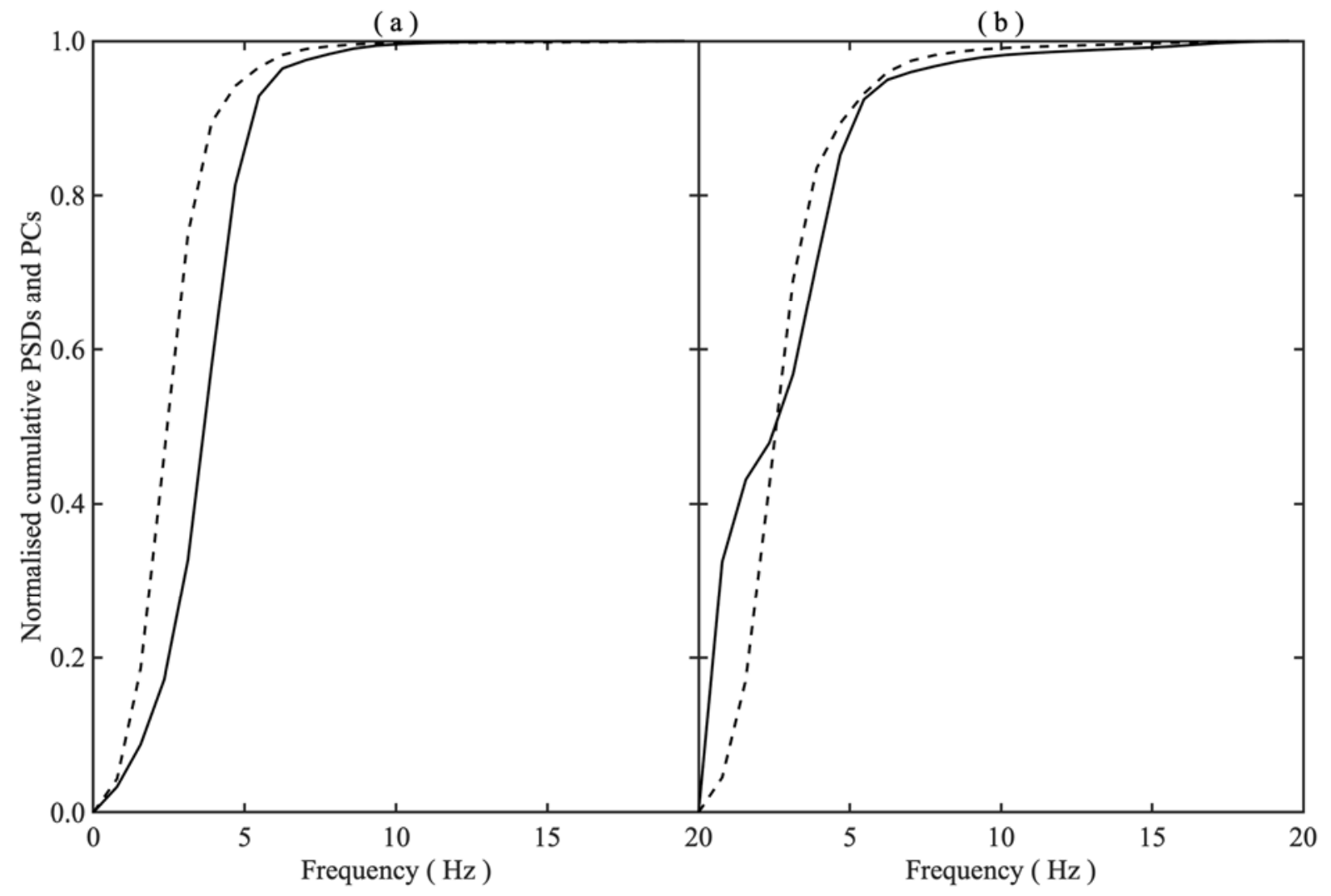

Figure 8 Individual (S9) normalised cumulative PSD of (a) G11 $c P S D_{G_{11}}(f)$ at 0.125 $\mathrm{ms}^{-2}$ r.m.s. (solid line) and $1.0 \mathrm{~ms}^{-2}$ r.m.s. (broken line), and (b) PC1 $c P S D_{P C 1}(f)$ at 0.125 $\mathrm{ms}^{-2}$ r.m.s. (solid line) and $1.0 \mathrm{~ms}^{-2}$ r.m.s. (broken line). 


\begin{tabular}{|c|c|c|c|c|}
\hline \multicolumn{3}{|c|}{$\begin{array}{l}\text { 1. Generate the characteristic spectral density matrix } G \text {, by } \\
\text { computing the nine components of CSDs and PSDs. } G \text { is } \\
\text { defined by Equation (3). }\end{array}$} & $\begin{array}{l}\text { Figure } 3 \mathrm{c}, \mathrm{f} \\
\text { Figure } 4 \mathrm{c}, \mathrm{f}\end{array}$ & \multirow{2}{*}{$\begin{array}{l}\text { 6. Compute the normalized } \\
\text { cumulative PSDs and their } \\
\text { differences, Equations (7-10). }\end{array}$} \\
\hline \multirow{2}{*}{\multicolumn{3}{|c|}{$\begin{array}{l}\text { 2. Apply eigen decomposition to find the scalar diagonal } \\
\text { matrix } D \text { containing the eigenvalue decomposition at each } \\
\text { frequency as in Equation (4). }\end{array}$}} & & \\
\hline & & & $\begin{array}{l}\text { Figure } 4 \text { a, d } \\
\text { Figure } 5\end{array}$ & \multirow[t]{4}{*}{ Figure 8} \\
\hline & & \\
\hline \multicolumn{3}{|c|}{$\begin{array}{l}\text { 3. Conduct singular value decomposition (SVD) to calculate } \\
\text { the matrices } U, S \text { and } V \text { at each frequency as in Equation (5). }\end{array}$} & Figure $6 \mathrm{a}, \mathrm{b}$ & \\
\hline 5 & & & & \\
\hline $\begin{array}{l}\text { 4. Use the } U \text { and } S \text { matrices } \\
\text { and PSD components of the } \\
G \text { matrix to compute virtual } \\
\text { coherences at each frequency } \\
\text { as in Equation (6). }\end{array}$ & $\begin{array}{l}\text { Figure } 6 \mathrm{c}, \mathrm{d}, \mathrm{e}, \mathrm{f} \\
\text { Figure } 7\end{array}$ & \multicolumn{2}{|c|}{$\begin{array}{l}\text { 5. Reconstruct } G \text { using the } \\
\text { modified } S \text { matrix, by using } \\
\text { one of the three singular } \\
\text { values and setting the others } \\
\text { to zero, and the existing } U \\
\text { and } V \text { matrices defined by } \\
\text { Equation (5). }\end{array}$} & $\begin{array}{l}\text { Figure } 3 \mathrm{~b}, \mathrm{e} \\
\text { Figure } 4 \mathrm{~b}, \mathrm{e}\end{array}$ \\
\hline
\end{tabular}

Figure 9 The signal processing workflow with reference to results. 